\title{
Rapid Simulation of a Hypersonic Vehicle Through Singular Value Decomposition
}

\author{
Ryan J. Klock ${ }^{*}$ and Carlos E.S. Cesnik ${ }^{\dagger}$ \\ University of Michigan, Ann Arbor, MI 48109-2140
}

\begin{abstract}
A reduced order model based on singular value decomposition and correlation is developed to capture the nonlinear dynamics of a hypersonic vehicle in flight. A set of training samples in state space form are collected using the complex step method. These samples are used to identify a set of ordered bases which describe the variation of the state matrices and state rates. Surrogate functions are fit to the coefficients of these bases to approximate the training samples and predict the state matrices outside of the training set. The dynamics of the system may then be rapidly simulated, provided the training set sufficiently populates the state space. This approach is first applied to a spring-mass-damper system with variable nonlinearity and number of degrees of freedom to determine training sample size and surrogate function orders which produce stable and accurate time simulation. A representative hypersonic vehicle is then considered to demonstrate the potential of this approach for rapid simulation of hypersonic flight.
\end{abstract}

\section{Introduction}

$\mathrm{H}$ IGH speed aircraft, particularly hypersonic vehicles, operate in a high energy environment characterized by strong fluid, thermal, and structural interactions. Due to a lack of ground test facilities which can generate the high energy environment of interest in aerothermoelastically scaled models, the primary focus for hypersonic vehicle design and basic evaluation must be through analytical and computational simulations. Current computational research efforts have focused on either improving particular physics model fidelity with limited discipline interactions due to a high computational cost, or including many discipline interactions using very simple models. Thus, there is a wide middle-ground between the low-fidelity, high-interaction and high-fidelity, lowinteraction modeling regimes that has yet to be considered, but is critical to the development of high speed systems.

To address the model regime gap, a simulation framework has been developed by the authors, named the University of Michigan High Speed Vehicle (UM/HSV) code. Within the UM/HSV code is a collection of reducedorder models (ROMs) which account for unsteady aerodynamics, ${ }^{1,2}$ aeroheating,${ }^{3,4}$ thermodynamics, ${ }^{4,5}$ thermoelastic coupling, ${ }^{6}$ and structural elastic dynamics ${ }^{6}$ with variable fidelities which can quickly and accurately perform $n$ degree of freedom flight simulations of full vehicles over entire flight trajectories. Real-time simulation has not yet been achieved but is required for hardware-in-the-loop trials. A possible method of accelerating the rate of simulation would be to express the equations of motion in a linear state space form in order to exploit vectorization in computer processors. The nonlinear nature of aeroheating, thermoelastic coupling, and supersonic aerodynamics makes this form challenging. ${ }^{7}$

In the past, methods of combining ROM techniques and linear parameter-varying (LPV) models have often begun with already determined state spaces with many degrees of freedom and sought to reduce the number of degrees of freedom while retaining the dominant behavior of the system in order to produce control laws. Such methods include modal reduction, ${ }^{8}$ balanced realization and truncation, ${ }^{8,9}$ Krylov methods, ${ }^{10}$ and others. ${ }^{11,12}$ It is not uncommon to use multiple techniques in combination when considering systems of very high order, such as in aeroelastic analyses of flexible aircraft. A recent example has been the simulation of the X-56A flight model where the influence of the airspeed and fuel weight was considered. ${ }^{12}$ In that work, a collection of state space matrices was first reduced by a combination of regular state truncation, modal reduction, and balanced truncation from 180 to 21 states. However, in this reduction process, the final 21 states did not have consistent meaning for all state spaces. To remedy this, a common subspace was determined from which all the state space matrices could be recovered. Matrices for state spaces outside of the original collection were determined through linear interpolation between nearest neighbor samples and shown to correctly predict the frequency response of most retained states.

\footnotetext{
* Graduate Research Assistant, Department of Aerospace Engineering, Student Member AIAA, rjklock@umich.edu

${ }^{\dagger}$ Professor, Department of Aerospace Engineering, AIAA Fellow, cesnik@umich.edu
}

American Institute of Aeronautics and Astronautics 
A different model reduction technique was developed by Carlson et al. ${ }^{13}$ in which the partial differential equations (PDEs) of motion for an F-16 similar aircraft were generalized using proper orthogonal decomposition (POD) modes derived from high-fidelity flight simulations of the maneuvering aircraft. The PDEs considered contained both linear and bi-linear terms in order to model some nonlinear behaviors including post-stall and aeroelasticity effects. Comparison of the reduced model and Kestrel flight simulation results showed good agreement, but with some loss of high-frequency dynamics.

For the study presented in this paper, a reduced order model (ROM) based on singular value decomposition (SVD) and correlation is developed to estimate the matrices which represent a nonlinear dynamic system in a state space form and state rates directly. The state spaces are updated while on-line, during the integration of the equations of motion, so an emphasis is placed on the speed of the ROM as well as accuracy. Training and testing samples are drawn from a pseudo-random Latin hypercube sampling (LHS) of system rates and state space matrices. Time simulations using full-order solutions and the SVD ROM are compared and conclusions drawn on the speed, accuracy, and feasibility of this approach.

\section{Theoretical Development}

The process to develop and train an SVD ROM for state space representations of nonlinear systems is overviewed. It begins by outlining how system dynamics may be expressed in state space, followed by how a state space representation may be approximated from an arbitrary system model. Construction and training of the SVD ROM is then described.

\section{A. State Space Representation}

A common approach to modeling system dynamics is with a state space representation consisting of a state matrix $A(x)$ and input matrix $B(x)$, which when multiplied by the state vector $x(t)$ and input vector $u(t)$ and summed, yields the state derivative vector $\dot{x}(t)$,

$$
\dot{x}(t)=A(x) x(t)+B(x) u(t) .
$$

For simple systems, $A$ and $B$ are often assumed to be time-invariant and independent of $x$ for small perturbations which reduces (1) to a linear system of equations. However, for a maneuvering hypersonic vehicle, the perturbations of $x$ may not be small and easily span a nonlinear regime. Thus, the dependence of $A$ and $B$ on $x$ must be captured for an accurate representation of the vehicle dynamics.

\section{B. Approximation of the State and Input Matrices}

Before construction of a state space ROM may begin, a set of sample state space matrices $A$ and $B$ must be obtained for the vehicle for different values of states $x$. Consider the state rates $\dot{x}$ to be an arbitrary function of the states

$$
\dot{x}=f(x, u) .
$$

An efficient and accurate method for determining the sensitivity of a function $f$ was developed in Ref. [14] by adding a small imaginary perturbation to each real state in that system in sequence. The derivative of the system near the location in real state space may be approximated by

$$
\frac{\partial f}{\partial x_{k}} \approx \frac{\operatorname{Im}\left[f\left(x+i h_{k}, u\right)\right]}{h_{k}},
$$

where $x_{k}$ is the $k^{\text {th }}$ state in $x, i$ is the imaginary number $\sqrt{-1}$, and $h_{k}=\left[0, \ldots, 0, h_{k}, 0, \ldots, 0\right]^{T}$ is the perturbation to the imaginary $k^{\text {th }}$ state. The state matrix $A$ is then

$$
A=\left[\begin{array}{llll}
\frac{\partial f}{\partial x_{1}} & \frac{\partial f}{\partial x_{2}} & \cdots & \frac{\partial f}{\partial x_{n}}
\end{array}\right] .
$$

The input matrix $B$ may be similarly constructed by adding imaginary perturbations to the input vector $u$. 


\section{Singular Value Decomposition and Correlation}

The next step is based on the work of Lillian, McDaniel, and Morton. ${ }^{15}$ Once a number of samples of $A$ and $B$ have been collected, each sample is combined into a single matrix by rearranging the entries $A$ and $B$ into columns, which then become the columns of a sample matrix

$$
S=\left[\begin{array}{cccc}
A_{1,1}\left(x^{(1)}\right) & A_{1,1}\left(x^{(2)}\right) & \cdots & A_{1,1}\left(x^{(s)}\right) \\
A_{1,2}\left(x^{(1)}\right) & A_{1,2}\left(x^{(2)}\right) & \cdots & A_{1,2}\left(x^{(s)}\right) \\
\vdots & \vdots & & \vdots \\
A_{1, n}\left(x^{(1)}\right) & A_{1, n}\left(x^{(2)}\right) & \cdots & A_{1, n}\left(x^{(s)}\right) \\
A_{2,1}\left(x^{(1)}\right) & A_{2,1}\left(x^{(2)}\right) & \cdots & A_{2,1}\left(x^{(s)}\right) \\
\vdots & \vdots & & \vdots \\
A_{n, n}\left(x^{(1)}\right) & A_{n, n}\left(x^{(2)}\right) & \cdots & A_{n, n}\left(x^{(s)}\right) \\
B_{1,1}\left(x^{(1)}\right) & B_{1,1}\left(x^{(2)}\right) & \cdots & B_{1,1}\left(x^{(s)}\right) \\
\vdots & \vdots & & \vdots \\
B_{n, m}\left(x^{(1)}\right) & B_{n, m}\left(x^{(2)}\right) & \cdots & B_{n, m}\left(x^{(s)}\right)
\end{array}\right],
$$

where $A_{i, j}\left(x^{(k)}\right)$ is the $i, j$ entry of matrix $A\left(x^{(k)}\right)$ which is the state matrix $A$ for the $k^{\text {th }}$ sample state vector $x^{(k)}$ for a total of $s$ samples. Similar for $B_{i, j}\left(x^{(k)}\right)$ for $n$ states and $m$ inputs. The sample state vectors are also combined into sample point matrices $d$. As will become evident later in this paper, it is advantageous to consider various permutations of the sample states to form a ROM which can capture nonlinearity in the state space. This work considers complete polynomials of the states such that

$$
\begin{aligned}
& d_{\text {constant }}=\left[\begin{array}{cccc}
1 & 1 & \cdots & 1 \\
\vdots & \vdots & & \vdots \\
1 & 1 & \cdots & 1
\end{array}\right]_{n \times s}, \\
& d_{\text {linear }}=\left[\begin{array}{cccc}
1 & 1 & \cdots & 1 \\
\vdots & \vdots & & \vdots \\
x_{1}^{(1)} & x_{1}^{(2)} & \cdots & x_{1}^{(s)} \\
\vdots & \vdots & & \vdots \\
x_{n}^{(1)} & x_{n}^{(2)} & \cdots & x_{n}^{(s)}
\end{array}\right]_{(2 n) \times s}, \\
& d_{\text {quadratic }}=\left[\begin{array}{cccc}
1 & 1 & \cdots & 1 \\
\vdots & \vdots & & \vdots \\
x_{1}^{(1)} & x_{1}^{(2)} & \cdots & x_{1}^{(s)} \\
\vdots & \vdots & & \vdots \\
x_{1}^{(1)} x_{1}^{(1)} & x_{1}^{(2)} x_{1}^{(2)} & \cdots & x_{1}^{(s)} x_{1}^{(s)} \\
x_{1}^{(1)} x_{2}^{(1)} & x_{1}^{(2)} x_{2}^{(2)} & \cdots & x_{1}^{(s)} x_{2}^{(s)} \\
\vdots & \vdots & & \vdots \\
x_{n}^{(1)} x_{n}^{(1)} & x_{n}^{(2)} x_{n}^{(2)} & \cdots & x_{n}^{(s)} x_{n}^{(s)}
\end{array}\right]_{\left(2 n+n^{2} / 2+n / 2\right) \times s}
\end{aligned}
$$


and so on, where $x_{n}^{(s)}$ is the $n^{\text {th }}$ entry of the $s^{\text {th }}$ sample. The sample point matrices do not contain duplicate nonlinear states since $x_{i} x_{j}$ is equivalent to $x_{j} x_{i}$. Sample point matrix dimensions are noted in the subscript of each matrix.

Using singular value decomposition, the sample matrix $S$ may be expressed as

$$
S=U \Sigma V^{T}
$$

where $U$ is a square matrix whose columns are the left singular vectors of $S, \Sigma$ is a rectangular diagonal matrix of the singular values ranked in descending magnitude, and $V$ is a square matrix whose columns are the right singular vectors. Due to the arrangement of the samples in matrix $S, U$ may be considered as a set of normalized basis matrices which can be used to construct the state space matrices $A$ and $B$, whose magnitudes in each of the samples is given by $\Sigma V^{T}$. Furthermore, $\Sigma$ gives the relative importance of each of the basis matrices in reconstructing the sample matrix. Provided the samples adequately populate the state space, then the bases of the sample matrices may also be used to describe the state space as a whole. If there are a large number of states which result in a large number of basis matrices, then truncation of the bases may be performed by removing the least important bases according to the singular values.

The next step is to relate the right singular vectors of $V$ to the sample points of $d$. A coefficient matrix $R$ is defined using a least squares approach such that

$$
d^{T} R=V,
$$

and determined by

$$
R=\left(d d^{T}\right)^{-1} d V
$$

Now, given any new state vector $\tilde{x}$ expressed as a vector of state polynomials $\tilde{d}$, an approximate sample matrix $\tilde{S}$ may be constructed via as

$$
\tilde{S}=U \Sigma R^{T} \tilde{d}
$$

which contains the entries of the state matrices $A$ and $B$ corresponding to $\tilde{x}$.

Determining the state rates $\dot{x}$ in (1) during integration of the equations of motion is then a 2-step process. In step 1 , the state matrices are determined from the SVD ROM, followed by step 2, where they are multiplied by the state vector $x$. It is also possible to use the SVD ROM to estimate the state rates $\dot{x}$ in a 1-step process by replacing the columns of matrix $S$ in (5) with the $\dot{x}$ vectors corresponding to each sampled state $x$, i.e.,

$$
S=\left[\begin{array}{llll}
\dot{x}\left(x^{(1)}\right) & \dot{x}\left(x^{(2)}\right) & \cdots & \dot{x}\left(x^{(s)}\right)
\end{array}\right] .
$$

During integration of the equations of motion, the state rates $\dot{x}$ are then determined in directly from the SVD ROM without the need to consider the state matrices.

\section{Reduced-Order Model Training}

The process of collecting state samples, training the SVD ROM, and determining if the model is complete is outlined in Figure 1. It begins with the definition of states and their respective limits. An LHS ${ }^{16}$ of the states is considered and state space representations of the system under the sampled conditions are estimated using the complex-step method ${ }^{14}$ described previously. The $s$ samples are compiled into the sample matrix $S$ and divided into two groups of $m$ and $k$ samples. The first $m$-samples for training the SVD ROM while the second is $k$-samples for testing the predictive accuracy of the trained ROM. The $m$-samples enter the ROM training function where the decomposition and surrogate fitting are carried out. The resulting ROMs are then evaluated for accuracy using normalized root mean square error (NRMSE) and maximum error $\left(\mathrm{L}_{\infty}\right)$ metrics for the $k$-testing samples. If the most accurate SVD ROM presents an error below some user defined tolerance, the SVD ROM is completed and ready for use as a surrogate for the higher fidelity reference model. If the error tolerance is exceeded, then additional samples are selected using LHS and the training process is repeated. 


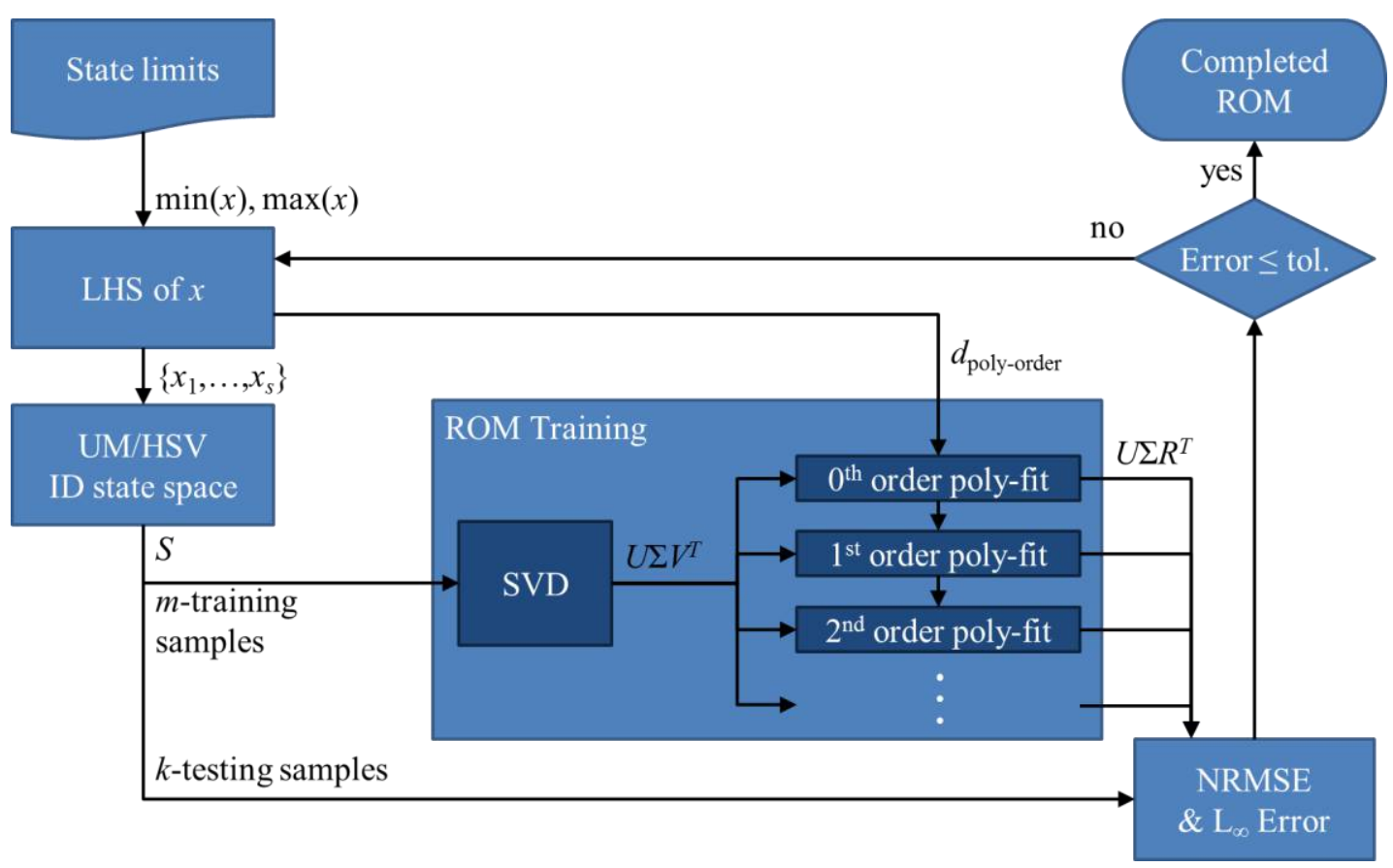

Figure 1: Outline of the SVD ROM training process

\section{Numerical Studies}

The method described in this work is applied to two test cases. The first is a nonlinear-spring, mass, and damper system which is used to determine the sample set size required to capture the dynamics of a system given its order of nonlinearity and number of degrees of freedom. Then, this information is used to train an SVD ROM to capture the behavior of a representative hypersonic vehicle.

\section{A. Test Cases}

\section{Nonlinear-Spring, Mass, and Damper}

The nonlinear-spring, mass, and damper system is shown in Figure 2. With this system, the number of degrees of freedom could be easily varied by chaining any number of masses together and the nature of the nonlinearity can be varied by choosing any of the stiffness, damping, and/or mass coefficients to be a function of displacement or time. For this study, the masses and damping coefficient were held constant while the stiffnesses were polynomial function of displacement ranging from $1^{\text {st }}$ to $6^{\text {th }}$ order. These coefficients are given in Table 1.

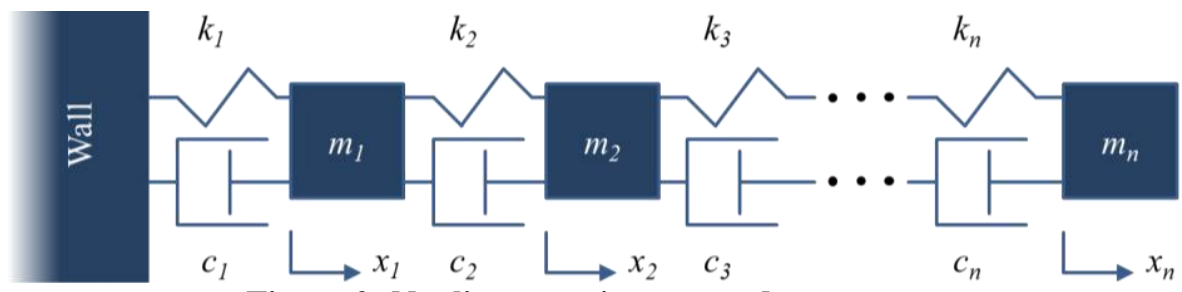

Figure 2: Nonlinear-spring, mass, damper system

Table 1: Nonlinear-spring, mass, damper coefficients $(i=1 \ldots n)$

\begin{tabular}{cc} 
Symbol & Value \\
\hline$m_{i}$ & 10.0 \\
$c_{i}$ & 0.1 \\
$k_{i}$ & $\left(x_{i}-x_{i-1}\right)^{p}$ \\
$x_{0}$ & 0
\end{tabular}

n: total number of spring, mass, and damper groups in chain

$i$ : index of spring, mass, and damper group in chain p: order of spring stiffness polynomial

5

American Institute of Aeronautics and Astronautics 
The state space representation of this system is given by

$$
\left[\begin{array}{c}
\dot{x}_{1} \\
\dot{x}_{2} \\
\vdots \\
\dot{x}_{n} \\
\ddot{x}_{1} \\
\ddot{x}_{2} \\
\vdots \\
\ddot{x}_{n}
\end{array}\right]=\left[\begin{array}{cccccccc}
0 & 0 & \cdots & 0 & 1 & 0 & \cdots & 0 \\
0 & 0 & \cdots & 0 & 0 & 1 & \cdots & 0 \\
\vdots & \vdots & \ddots & \vdots & \vdots & \vdots & \ddots & \vdots \\
0 & 0 & \cdots & 0 & 0 & 0 & \cdots & 1 \\
\frac{-k_{1}}{m_{1}}-\frac{k_{2}}{m_{1}} & \frac{k_{2}}{m_{1}} & \cdots & 0 & \frac{-c_{1}}{m_{1}}-\frac{c_{2}}{m_{1}} & \frac{c_{2}}{m_{1}} & \cdots & 0 \\
\frac{k_{2}}{m_{2}} & \frac{-k_{2}}{m_{2}}-\frac{k_{3}}{m_{2}} & \cdots & 0 & \frac{c_{2}}{m_{2}} & \frac{-c_{2}}{m_{2}}-\frac{c_{3}}{m_{2}} & \cdots & 0 \\
\vdots & \vdots & \ddots & \frac{k_{n}}{m_{n-1}} & \vdots & \vdots & \ddots & \frac{c_{n}}{m_{n-1}} \\
0 & 0 & \frac{k_{n}}{m_{n}} & \frac{-k_{n}}{m_{n}} & 0 & 0 & \frac{c_{n}}{m_{n}} & \frac{-c_{n}}{m_{n}}
\end{array}\right]\left[\begin{array}{c}
x_{1} \\
x_{2} \\
\vdots \\
x_{n} \\
\dot{x}_{1} \\
\dot{x}_{2} \\
\vdots \\
\dot{x}_{n}
\end{array}\right],
$$

and may be used to directly check the accuracy of the SVD ROM's estimates.

\section{Sample Hypersonic Vehicle}

The second test case is a representative hypersonic vehicle configuration modified from the Initial Concept 3.X (IC3X) design established by Witeof and Neergaard ${ }^{17}$ using the PASS code suite ${ }^{18}$ and shown in Figure 3.
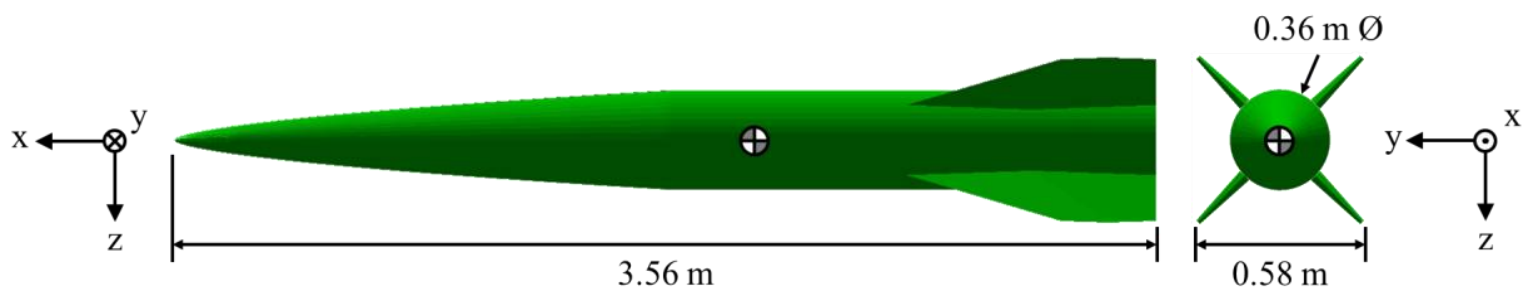

Figure 3: IC3X representative hypersonic vehicle configuration

The body is axisymmetric with four all-movable diamond airfoil fins for stability and control. The nose tip is a 10-mm diameter hemisphere tangent-transitioned to a power-law forebody. The aft body is a circular cylinder. Inertial properties are given in Table 2.

Table 2: Inertial properties of the IC3X

\begin{tabular}{ccc} 
Property & Fully Fueled & Reserve Fuel Only \\
\hline Center of gravity $(\mathrm{m})$ & $2.09,0,0$ & $2.06,0,0$ \\
Mass $(\mathrm{kg})$ & 375 & 307 \\
$\mathrm{I}_{\mathrm{xx}}\left(\right.$ about CG) $\left(\mathrm{kg}-\mathrm{m}^{2}\right)$ & 9.42 & 7.37 \\
$\mathrm{I}_{\mathrm{yy}}$ (about CG) $\left(\mathrm{kg}-\mathrm{m}^{2}\right)$ & 345 & 338 \\
$\mathrm{I}_{\mathrm{zz}}$ (about CG) $\left(\mathrm{kg}-\mathrm{m}^{2}\right)$ & 345 & 338
\end{tabular}

Elastic deformation of the vehicle is modeled using free-vibration mode shapes determined from analysis of a finite element model. ${ }^{17}$ For the work presented in this paper, the first two bending modes of the vehicle were considered. These mode shapes are shown in Figure 4. Note that the two mode shapes are identical in form, with the difference that one is rotated $90^{\circ}$ about the $\mathrm{x}$-axis. 


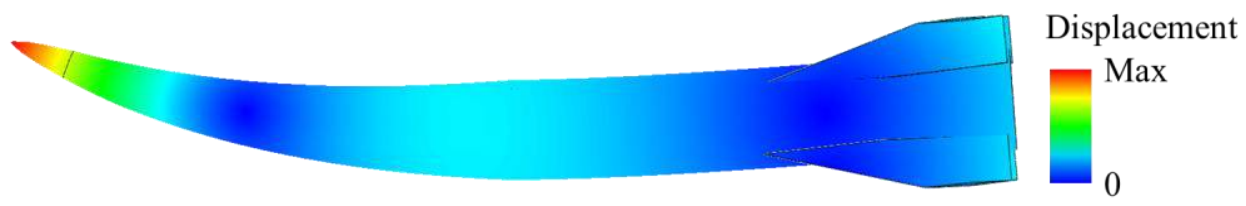

Figure 4: First lateral and longitudinal bending modes

Determining the state space representation of the IC $3 \mathrm{X}$ is not as straight-forward as the spring, mass and damper, but must be estimated from a full vehicle model. To do this, the University of Michigan High Speed Vehicle (UM/HSV) code was used and from which the state space matrices $A$ and $B$ were estimated. It also provides reference flight simulation against which to compare the SVD ROM solutions.

\section{B. Reduced-Order Model Stability}

To begin to understand the behavior and performance of the SVD ROM approach, the number of required samples to represent a system of given order and degrees of freedom was investigated using the nonlinear-spring, mass, and damper test case. The chain of masses was initially set to an equilibrium state with zero velocity and all masses at zero displacement. The $n^{\text {th }}$ mass was then displaced by 1, released, and the system dynamics integrated forward in time using the MATLAB ${ }^{19}$ ode 45 solver.

During testing, it was found that if a state exceeded the range of the training set, causing the SVD ROM to extrapolate, the states would often diverge toward infinity. This instability would frequently prevent numerical integration of the system dynamics. Insufficient range of the training set is typically a symptom of too sparse of a sampling of the state space and was remedied by increasing the number of training samples. An example of this unstable system dynamics due to under-sampling is shown in Figure 5.

To determine the boundary of the SVD ROM stability, the training set size $m$ was varied from 2 to $2^{14}$ by powers of 2 and the maximum number of stable degrees of freedom was determined for each case. This was carried out for systems whose spring constants were $2^{\text {nd }}, 4^{\text {th }}$, and $6^{\text {th }}$ powers of the elongation of the springs. The results are shown in Figure 6.

It can be seen by fitting power-law curves to the results how the number of stable degrees of freedom depend on the training set size and system order. It is apparent that greater the number of degrees of freedom and the greater the order of the system, then the greater the number of samples that will be needed to capture the behavior of that system for both the 2-step and 1-step SVD ROM approaches. The 1-step approach also requires more samples than the 2-step approach for a given number of degrees of freedom and system order. This is due to the fact that in the 1step approach, there is no second multiplication by the state vector $x$ after the SVD ROM is exercised. Thus the 1step approach is only capable of modeling one order less than the 2-step approach. The efficiency of the SVD ROM as additional training samples were added can be considered as

$$
\text { Efficiency }=m^{(-1 / n)} \text {. }
$$

In this form, the fewer the number of training samples $m$ required by the SVD ROM to capture the behavior of a system with $n$ degrees of freedom, and therefore a sample space of $n$ dimensions, then the closer the efficiency value approaches unity. From Figure 7 it can be seen that the efficiency of the training set tends to increase as the number of degrees of freedom increase. This means that for a system similar to the nonlinear-spring, mass, and damper chain posed in this work, where the dynamics of each state are similar, the training sample point density in the state space may be reduced as more degrees of freedom are considered. This is a typical feature of the LHS used in this work, as each additional sample strives to be far and equidistant from all previous samples in all dimensions to maximize the amount of useful information obtained. ${ }^{20}$ 
256 training samples
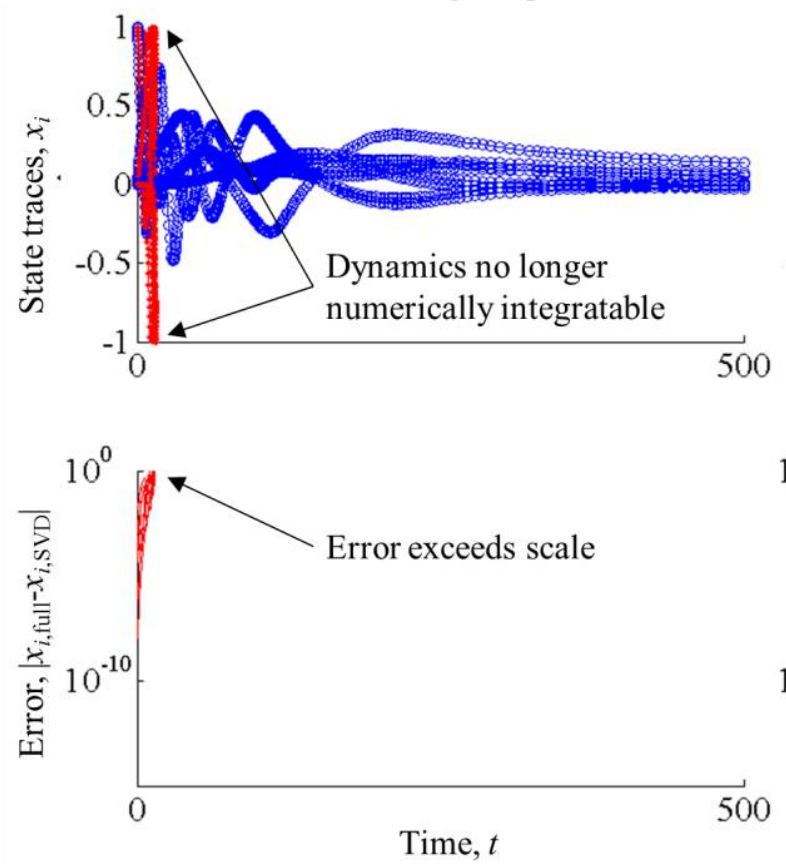

512 training samples
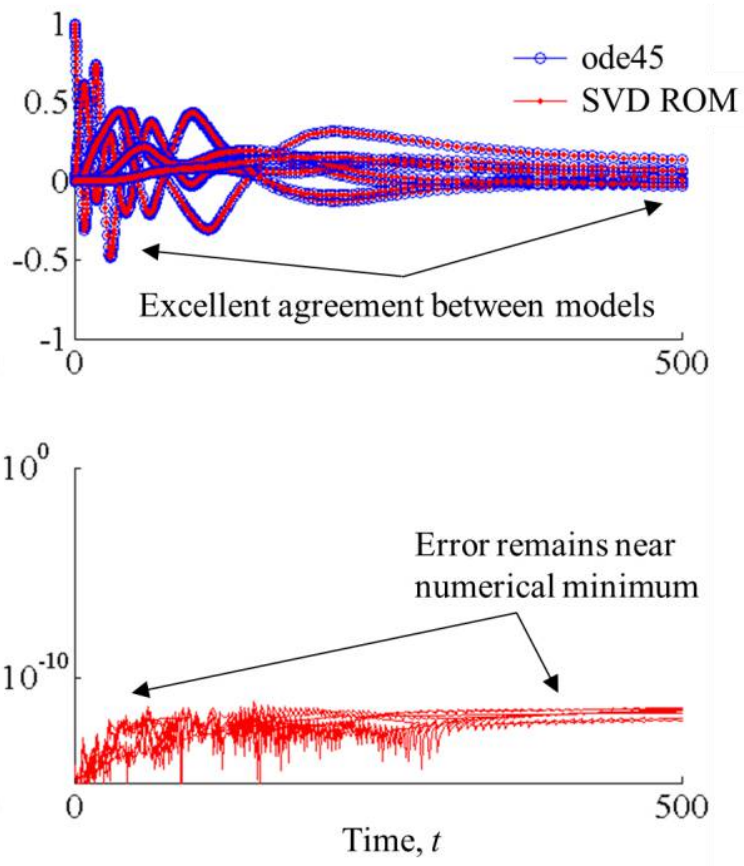

Figure 5: Example of SVD ROM instability due to under-sampled $4^{\text {th }}$ order, 8 degree-of-freedom nonlinearspring, mass, and damper case

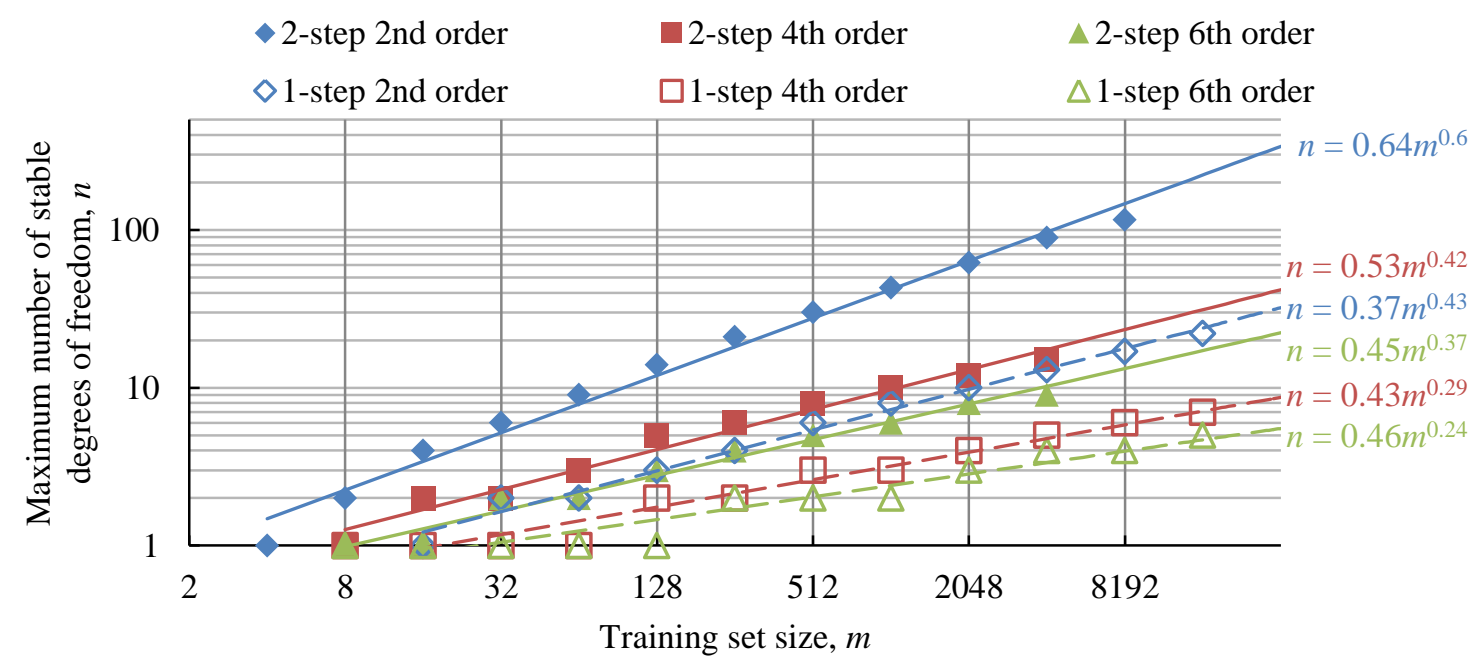

Figure 6: Stability boundary of the SVD ROM for various system orders 


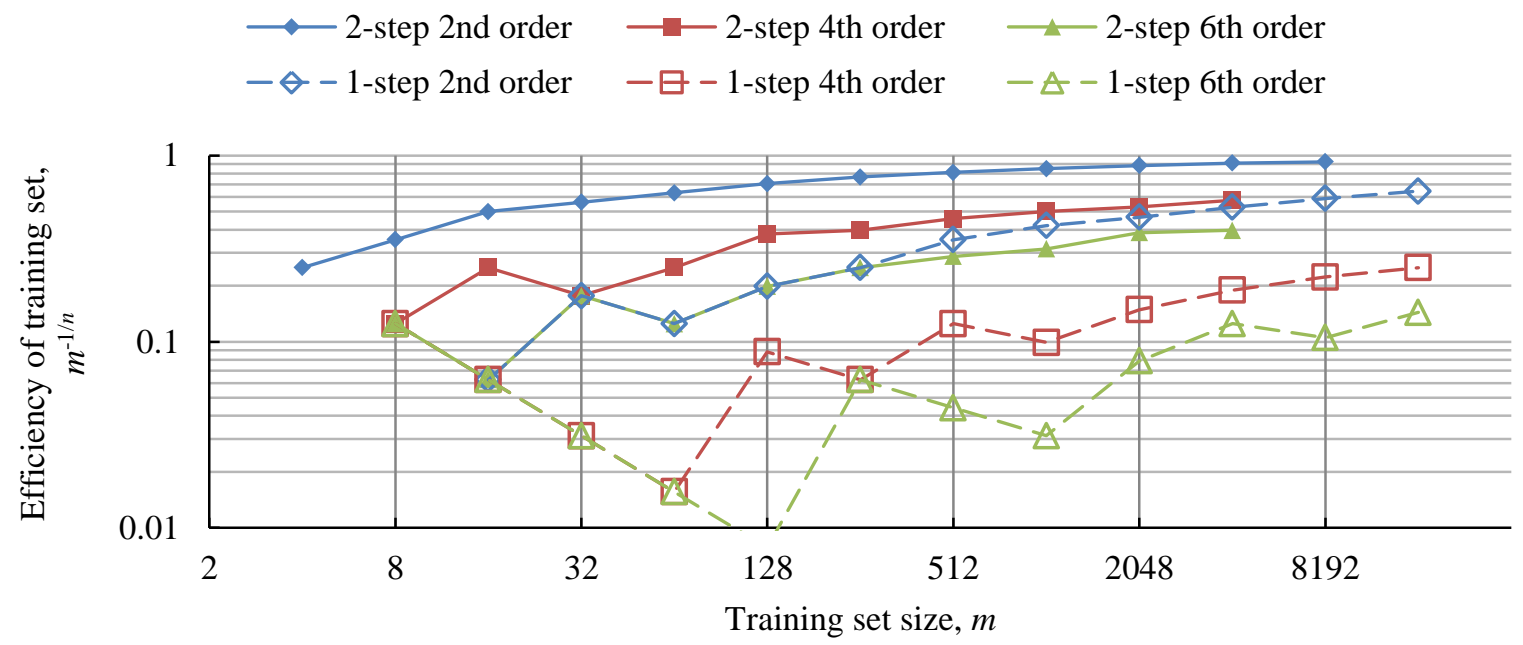

Figure 7: Increasing efficiency of training sample set with increasing number of degrees of freedom

\section{Application to the IC3X Vehicle}

To test the SVD ROM approach on a more realistic system, the AFRL IC3X vehicle dynamics were considered. In addition to the 12 rigid body states typical of 6-degree of freedom flight simulations and the 4 elastic states required to consider the 2 elastic degrees of freedom, the deflection angles of the 4 control fins were also included. The sampling ranges for each of these states for the experiments to follow are shown in Table 3 . A training set of $m=270,000$ samples was collected and a 1 -step, $4^{\text {th }}$ order SVD ROM trained with 20 retained bases. This training set size was selected by solving the power-curve fit to the data shown in Figure 6 for the $n=16$ independent degrees of freedom and rounding up. A time simulation using the SVD ROM was then compared to the UM/HSV code for a 1 second top-hat pitch-up maneuver in which the fins were deflected $1^{\circ}$ shown in Figure 8. The resulting state traces throughout the 3-second maneuver are shown in Figure 9 with the individual longitudinal states shown in Figure 10 and Figure 11 along with the normalized error of the SVD ROM according to

$$
\text { Normalized error }=\frac{\left|x_{i, \mathrm{HSV}}-x_{i, \mathrm{SVD}}\right|}{\max \left(\left|x_{i, \mathrm{HSV}}\right|\right)},
$$

where $x_{i}$ is a column matrix containing the trace of the $i^{\text {th }}$ state. For all longitudinal states, the SVD ROM solution matches very well with UM/HSV. A maximum error of $25.9 \%$ for the longitudinal bending rate $\dot{\eta}_{2}$ is shown during the initial deflection of the fins but recovers quickly to a mean error of $0.5 \%$ over the duration of the maneuver. Most profound is the difference in processing time. To consider this 3 second maneuver using a computer with an Intel Xeon E5-2650 2.0 GHz processor and $32 \mathrm{~GB}$ of memory, the UM/HSV code required 239.3 seconds while the SVD ROM required 17.2 seconds. This is nearly a 14 times increase in simulation speed increase. 
Table 3: State and input sample ranges for the IC3X vehicle SVD ROM training set

Description

Body axial velocity $(\mathrm{m} / \mathrm{s})$

Body lateral velocity $(\mathrm{m} / \mathrm{s})$

Body vertical velocity $(\mathrm{m} / \mathrm{s})$

Roll rate $(\%)$

Pitch rate $(\%)$

Yaw rate $(\%)$

Earth-body axial displacement (m)

Earth-body lateral displacement (m)

Earth-body vertical displacement (m)

Earth-body roll angle $\left(^{\circ}\right)$

Earth-body pitch angle $\left(^{\circ}\right)$

Earth-body yaw angle $\left({ }^{\circ}\right)$

Lateral bend amplitude

Longitudinal bend amplitude

Lateral bend rate $(1 / \mathrm{s})$

Longitudinal bend rate (1/s)

Fin 1 deflection $\left(^{\circ}\right)$

Fin 2 deflection $\left(^{\circ}\right)$

Fin 3 deflection $\left(^{\circ}\right)$

Fin 4 deflection $\left(^{\circ}\right)$

Symbol

$w$

$p$

\section{$q$}

$r$
$r$
$y$

$x$
$y$

\section{$z$}

q

$\theta$

$\psi$

$-0.6$

$\eta_{1} \quad-0.001$

$\eta_{2}$

$\dot{\eta}_{1}$

$\dot{\eta}_{2}$

$\delta_{1}$

$\delta_{2}$

$\delta_{3}$

$\delta_{4}$
Max.

$1744 \quad 1770$

$-0.01 \quad 0.01$

$-40 \quad 24$

$-0.6 \quad 0.6$

$-23 \quad 29$

$\begin{array}{ll}-0.6 & 0.6\end{array}$

$0 \quad 5310$

$-0.1 \quad 0.1$

$\begin{array}{ll}-6 & 9\end{array}$

-0.6 $\quad 0.6$

$-1.2 \quad 12$

0.6

0.001

0.001

0.1

$-0.1 \quad 0.1$

$0 \quad 1$

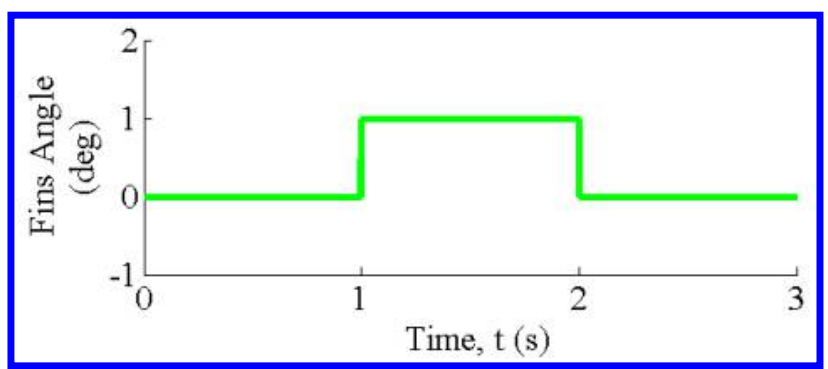

Figure 8: Fin deflection input signal
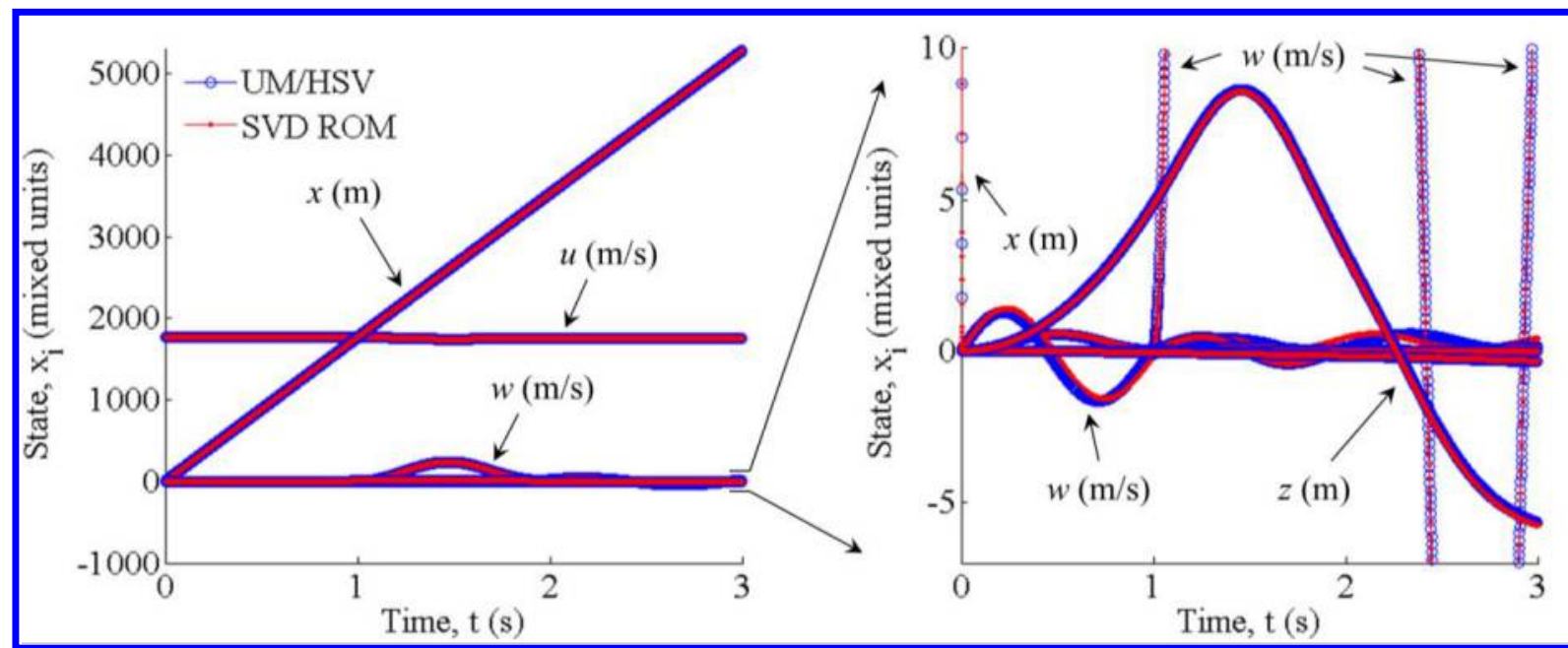

Figure 9: Comparison of UM/HSV and the 1-step, 4 "'-order SVD ROM with 20 retained bases during a 3second, 1-degree pitch maneuver

10

American Institute of Aeronautics and Astronautics 


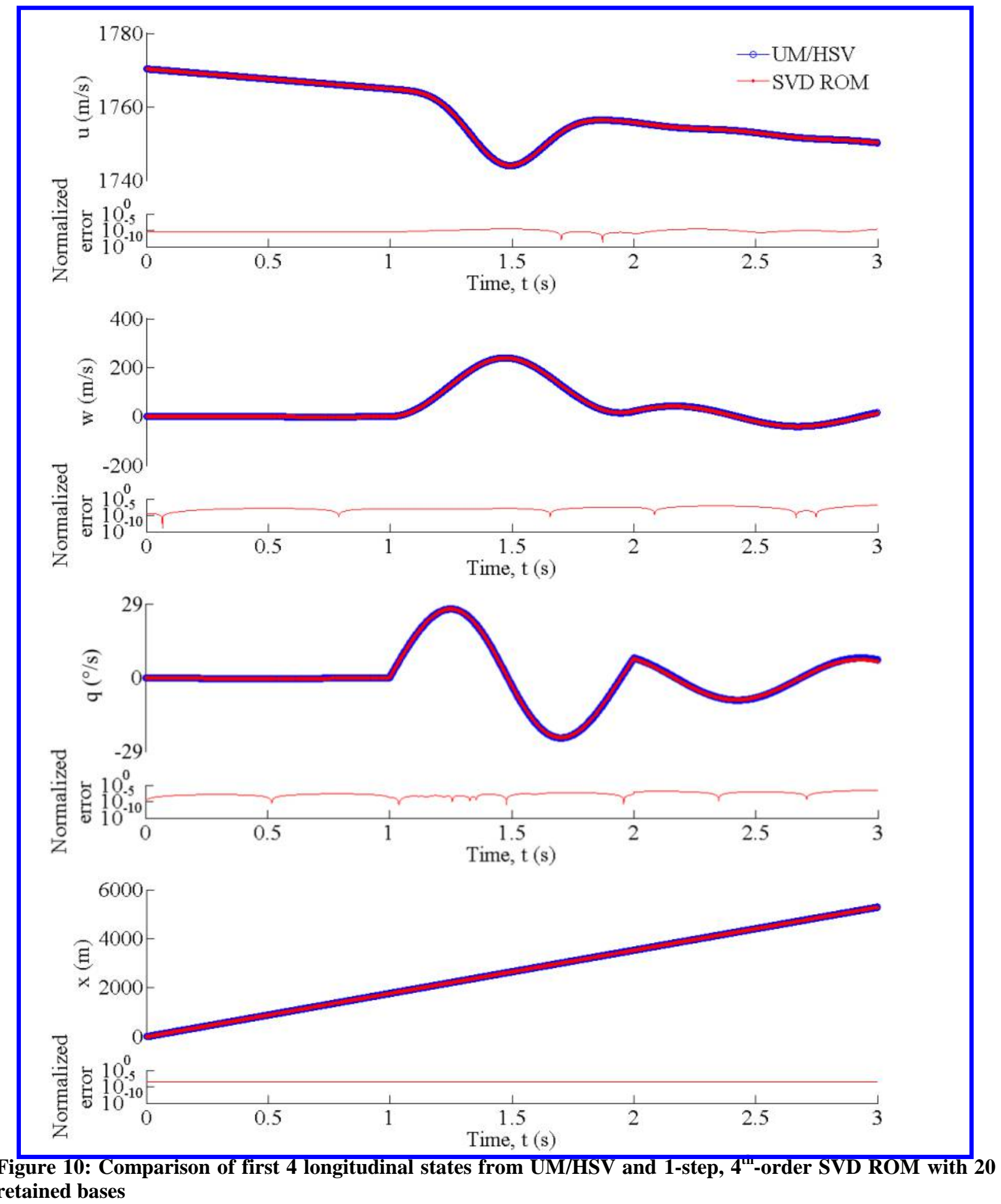



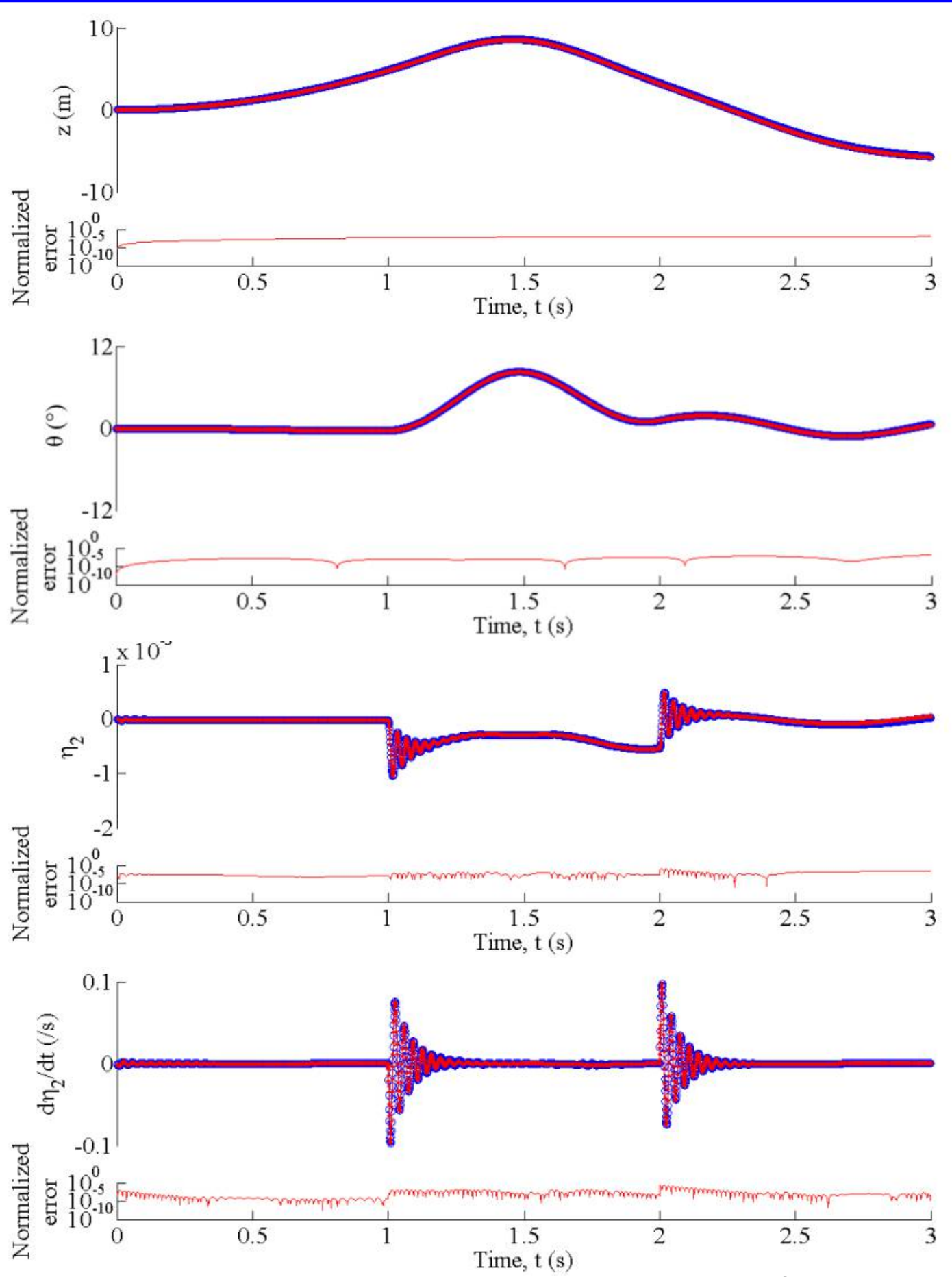

Figure 11: Comparison of last 4 longitudinal states from UM/HSV and 1-step, 4" -order SVD ROM with 20 retained bases 


\section{Singular Vector Truncation}

As discussed in section II.C, the singular values contained in $\Sigma$ offer a means of ranking the relative importance of the singular vectors $U$ in describing the singular space. In order to further decrease the processing time of the SVD ROM simulation, it may be possible to reduce the number of relevant singular vectors by truncating those corresponding to the lowest singular values. The number of dimensions in the singular space would then be reduced, simplifying the integration problem, while sacrificing as little accuracy as possible. ${ }^{5}$ To determine the effect of truncation on the solution accuracy, the IC3X 1 -step, $4^{\text {th }}$ order SVD ROM was retrained while retaining the $1^{\text {st }}$ through $20^{\text {th }}$ most important singular vectors. The root-mean-squared error of all 16 states was then determined at the final time step for the 1-degree pitch-up maneuver and is shown in Figure 12. The processing time required to integrate the maneuver's equations of motion is shown in Figure 13.

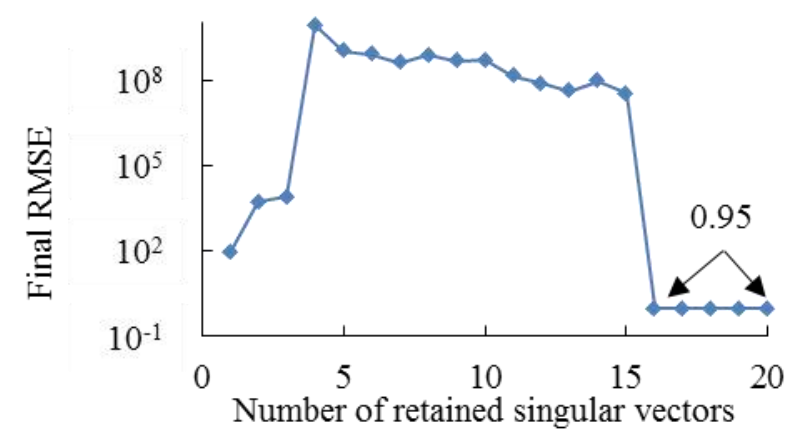

Figure 12: Error occurred due to truncation of the singular vectors

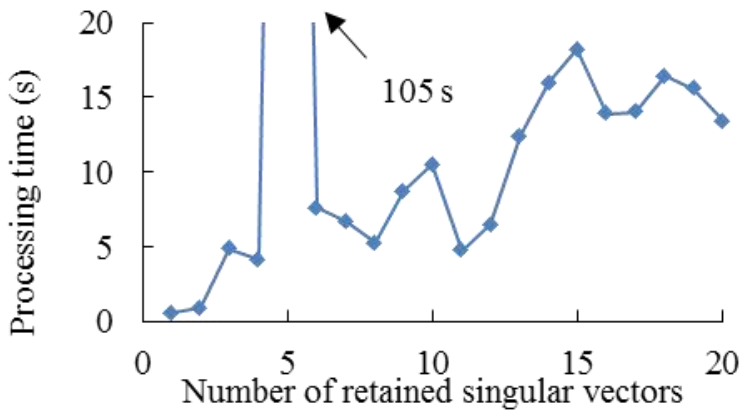

Figure 13: Processing time reduction due to truncation of the singular vectors

While retaining 1, 2, or 3 singular vectors appeared to provide a somewhat accurate solution using a small amount of processing time, this was largely due to the fact that the solution was numerically integratable, as shown in Figure 14. Retaining 4 to 15 vectors lead to solutions that increasingly matched the UM/HSV solution, but inevitably diverged and were unable to be integrated. The divergence of the SVD ROM estimated states from the UM/HSV solution was not unique to any single state, but rather would occur in all states simultaneously. This was a result of the SVD ROM expressing the states as combinations of singular vectors. As any one singular vector was forced to extrapolate because of a combination of states that was outside of the training set, then all state estimates would express divergent behavior similar to that observed in Figure 5. Retaining 16 or more vectors, matching the original number of degrees of freedom, resulted in accurate and stable solutions. Retaining more singular vectors typically required more processing time, but this trend was not closely followed as the ode 45 solver attempted to refine the time steps near highly dynamic periods.
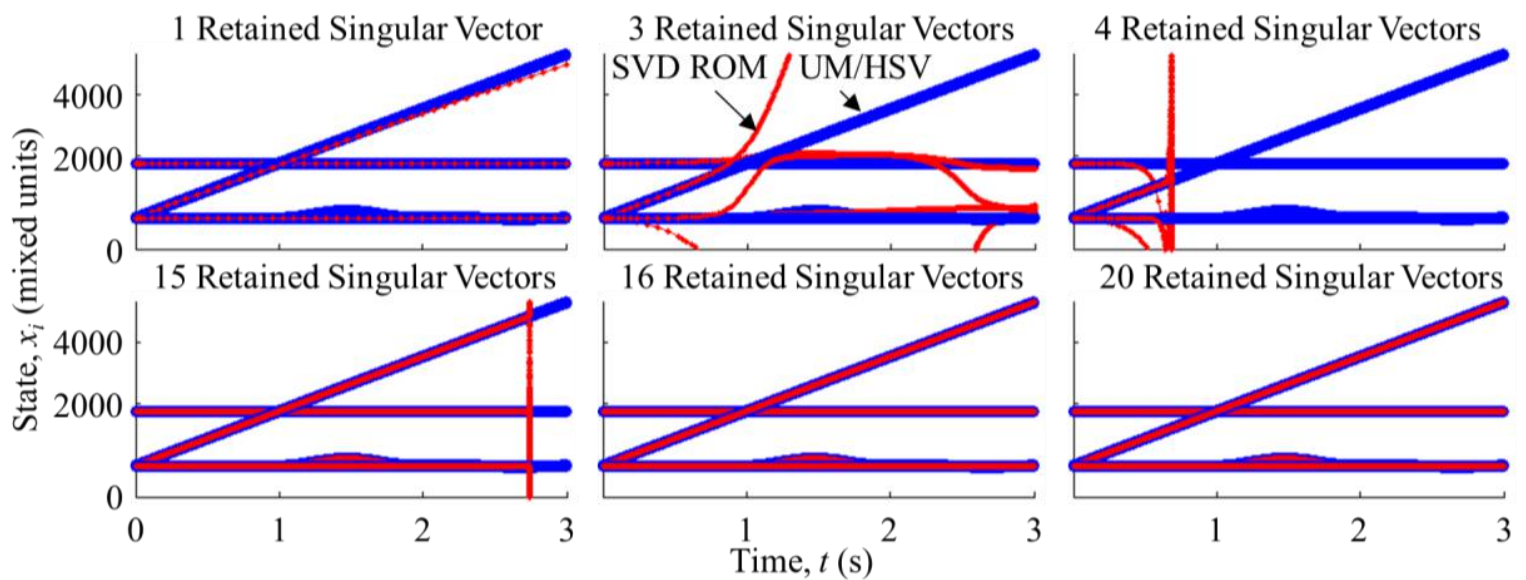

Figure 14: Comparison of the SVD ROM and UM/HSV solutions for a 3-second, 1-degree pitch maneuver using various numbers of retained singular vectors 


\section{E. Coefficient Matrix Compression}

Another approach to reduce the processing time of the SVD ROM is to consider the structure of the matrix $U \Sigma R^{T}$ in (12) for the 1-step approach and shown in Figure 15 for the IC3X 1-step, $4^{\text {th }}$-order model. Not all terms of the state polynomial significantly affect the state rates. This results in a great number of columns of $U \Sigma R^{T}$ containing only zeros or very small values. Neglecting columns whose maximum values are below some tolerance and forming a more compact matrix could reduce the number of operations required to integrate the system. By simply removing the zero columns, the number of entries can be reduced by more than half, from 28,640 to 11,264.

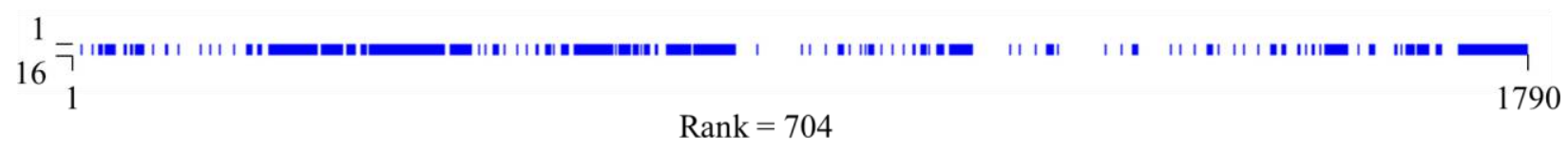

Figure 15: Non-zero entries of $U \Sigma R^{T}$ marked in blue for the IC3X, 1-step, $4^{\text {th }}$-order SVD ROM

Similar to the previous section, the RMSE at the end of the 3-second, 1-degree pitch-up maneuver was used to indicate the accuracy of the SVD ROM. A minimum magnitude tolerance varying from $10^{-10}$ to $10^{-5}$ was used to determine which columns of $U \Sigma R^{T}$ would be retained. The number of retained columns for a given tolerance, the RMSE after integration, and the processing time are shown in Figure 16. Using a low tolerance value (below $10^{-8}$ ) reduced processing time by $22 \%$ without increasing error when removing all unnecessary zeros. Between $10^{-8}$ and $3 \cdot 10^{-7}$, non-zero containing columns were removed which caused the error to increase slightly, but between $3 \cdot 10^{-7}$ and $10^{-6}$ solution error curiously decreased below the uncompressed solution. This could be due to removing coefficients which contained some numerical error from the SVD or fitting process, but merits further investigation before a final conclusion is reached regarding its cause. Beyond $10^{-6}$ the accumulated error during integration grew beyond the sample set range and caused the SVD ROM to extrapolate, which caused the solution to not be numerically integratable, as previously shown for with the nonlinear-spring, mass, and damper case.

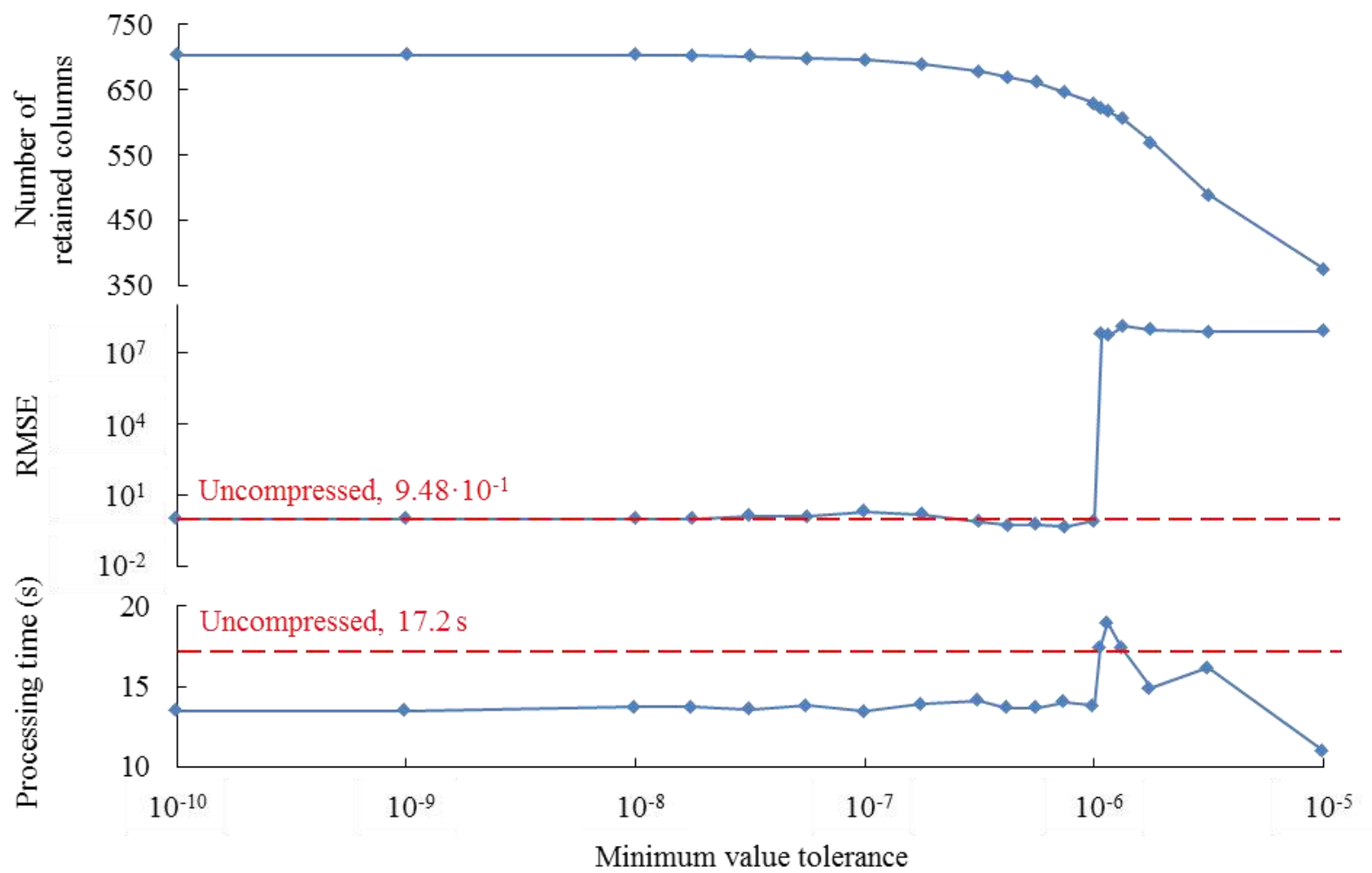

Figure 16: Performance of compressed SVD ROM 


\section{Conclusions}

A reduced-order model approach based on SVD and correlation has been developed and used to quickly simulate the dynamics of nonlinear systems. Two examples were chosen to further demonstrate the new method: a nonlinearspring, mass, and damper system and a representative hypersonic vehicle. State space and state rate samples were determined using the complex-step method for each system given a set of training states selected using LHS. Bases vectors where determined using SVD and polynomial functions up to $6^{\text {th }}$ order were fit as surrogates to the training samples expressed in the singular space. During integration of a system's equations of motion, the SVD ROM could either be used in a 2-step approach to estimate the state matrices $A$ and $B$ for a given state $x$, thus capturing nonlinear effects typically excluded from a state space representation, or in a 1-step approach to directly estimate the state rates $\dot{x}$.

While the SVD ROM method performed well when interpolating within the range of the training set and provided well behaved, accurate solutions during integration of the equations of motion. It was observed that extrapolation often resulted in divergent solutions with state values that tended toward infinity. This divergent behavior would also result from a too sparsely sampled state space. A nonlinear-spring, mass, and damper test case was used to determine the number of samples required to accurately represent a given number of states and nonlinearity. The dynamics of up to 116 states was captured with as few as 8192 training samples.

The SVD ROM method was then applied to the Initial Concept 3.X representative hypersonic vehicle with training samples obtained from the University of Michigan High Speed Vehicle code. A 3-second pitch-up maneuver was performed by deflecting the control fins by 1 degree for which the UM/HSV code required 239.3 seconds to simulate. Integration of the equations of motion using the SVD ROM required 17.2 seconds in the same computer, a nearly 14 fold speedup, with mean normalized error of approximately $0.5 \%$. Truncation of the singular vectors for additional simulation acceleration was investigated but did not yield improvement without inducing the divergent extrapolation behavior previously discussed. Compression of the $U \Sigma R^{T}$ matrix by removing small, maximum-valued columns moderately improved simulation speed and reduced the processing time for the maneuver down to 13.5 seconds, nearly an 18 fold speedup, without significantly increasing the solution error.

\section{Acknowledgements}

This work was sponsored by the Air force Research Laboratory (AFRL), Munitions Directorate, Eglin Air Force Base, Florida. The technical monitors are Drs. Crystal Pasiliao and Daniel Reasor. Opinions, interpretations, conclusions, and recommendations are those of the authors and are not necessarily endorsed by the United States Government.

\section{References}

$\checkmark$ McNamara, J. J., Crowell, A. R., Friedmann, P. P., Glaz, B., and Gogulapati, A., "Approximate Modeling of Unsteady Aerodynamics for Hypersonic Aeroelasticity," Journal of Aircraft, Vol. 47, No. 6, November 2010, pp. 1932-1945.

$\triangleright^{2}$ Zettl, D., Dreyer, E., Grier, B., McNamara, J. J., and Pasiliao, C. L., "Rapid Steady-State Pressure Prediction for Ultra High-Speed Vehicles," Proceedings of AIAA SciTech, 4-8 January 2016, San Diego, California, AIAA 2016-1323.

3 Crowell, A. R., McNamara, J. J., Kecskemety, K. M., and Goerig, T. W., "A Reduced Order Aerothermodynamic Modeling Framework for Hypersonic Aerothermoelasticity," Proceedings of the 51st AIAA/ASME/ASCE/AHS/ASC Structures, Structural Dynamics, and Materials Conference, 12-15 April 2010, Orlando, Florida, AIAA 2010-2969.

4 Klock, R., and Cesnik, C. E., "Nonlinear Thermal Reduced Order Models of a Hypersonic Vehicle," Proceedings of the 15th Dynamics Specialists Conference, 4-8 January 2016, San Diego, California, AIAA 2016-1322.

-5 Falkiewicz, N. J., and S. Cesnik, C. E., "Proper Orthogonal Decomposition for Reduced-Order Thermal Solution in Hypersonic Aerothermoelastic Simulations," AIAA Journal, Vol. 49, No. 5, May 2011, pp. 994-1009.

6 Falkiewicz, N. J., and Cesnik, C. E. S., "Enhanced Modal Solutions for Structural Dynamics in Aerothermoelastic Analysis," Proceedings of the 52nd AIAA/ASME/ASCE/AHS/ASC Structures, Structural Dynamics, and Materials Conference, 4-7 April 2011, Denver, Colorado, AIAA 2011-1963.

7 Klock, R. J., and Cesnik, C. E. S., "Aerothermoelastic Simulation of Air-Breathing Hypersonic Vehicles," Proceedings of the 55 $5^{\text {th }}$ AIAA/ASME/ASCE/AHS/ASC Structures, Structural Dynamics, and Materials Conference, 13-17 January 2014, National Harbor, Maryland, AIAA 2014-0149. 
$\checkmark 8$ Theis, J., Takarics, B., Pfifer, H., Balas, G. J., and Werner, H., "Modal Matching for LPV Model Reduction of Aeroservoelastic Vehicles," Proceedings of the AIAA Atmospheric Flight Mechanics Conference, 5-9 January 2015, Kissimmee, Florida, AIAA 2015-1686.

9 Hjartarson, A., Seiler, P. J., and Balas, G. J., "LPV Aeroservoelastic Control using the LPVTools Toolbox," Proceedings of the AIAA Atmospheric Flight Mechanics Conference, 19-22 August 2013, Boston, Massachusetts, AIAA 2013-4742.

-10 Poussot-Vassal, C., and Roos, C., "Flexible Aircraft Reduced-order LPV Model Generation from a Set of Largescale LTI Models," Proceedings of the 2011 American Control Conference, 29 June - 1 July 2011, San Francisco, California, pp. 745-750.

$\checkmark 11$ Adegas, F. D., Sønderby, I. B., Hansen, M. H., and Stoustrup, J., "Reduced-order LPV model of flexible wind turbines from high fidelity aeroelastic codes," Proceedings of the 2013 IEEE International Conference on Control Applications, 28-30 August 2013, Hyderabad, India, pp. 424-429.

12 Wang, Y., Song, H., Pant, K., Brenner, M. J., and Suh, P. M., "Model Order Reduction of Aeroservoelastic Model of Flexible Aircraft," Proceedings of the 57th AIAA/ASCE/AHS/ASC Structures, Structural Dynamics, and Materials Conference, 4-8 January 2016, San Diego, California, AIAA 2016-1222.

13 Carlson, H. A., and Verberg, R., "A Flight Simulator for Agile Fighter Aircraft and Nonlinear Aerodynamics," Proceedings of the 53rd AIAA Aerospace Sciences Meeting, 5-9 January 2015, Kissimmee, Florida, AIAA 20151506.

${ }^{14}$ Martins, J. R. R. A., Kroo, I. M., and Alonso, J. J., "An Automated Method for Sensitivity Analysis Using Complex Variables," Proceedings of the 38th Aerospace Sciences Meeting and Exhibit, 10-13 January 2000, Reno, Nevada, AIAA 2000-0689.

15 Lillian, C. S., McDaniel, D. R., and Morton, S. A., "An Efficient Method of Computing Maneuvering Aircraft Surface Loads Using CFD, Proper Orthogonal Decomposition, and System Identification," Proceedings of the 49th AIAA Aerospace Sciences Meeting, 4-7 January 2011, Orlando, Florida, AIAA 2011-1177.

- 16 Sacks, J., Welch, W. J., Mitchell, T. J., and Wynn, H. P., "Design and Analysis of Computer Experiments," Statistical Science, Vol. 4, No. 4, November 1989, pp. 409-423.

17 Witeof, Z. D., and Neergaard, L. J., "Initial Concept 3.0 Finite Element Model Definition," Air Force Research Laboratory, Rept. AFRL-RWWV-TN-2014-0013, Eglin AFB, FL, April 2014. (96ABW-2014-0141).

18 Pasiliao, C. L., Sytsma, M. J., Neergaard, L. J., Witeof, Z. D., and James.W., T., "Preliminary Aerothermal Structural Simulation," Proceedings of the 14th AIAA Aviation Technology, Integration, and Operations Conferance, 16-20 June 2014, Atlanta, Georgia, AIAA 2014-2292.

19 MathWorks, "MATLAB Documentation" Available: https://www.mathworks.com/help/, Accessed: 21 Oct. 2016.

-20 Manteufel, R., "Evaluating the Convergence of Latin Hypercube Sampling," Proceedings of the 41 AIAA/ASME/ASCE/AHS/ASC Structures, Structural Dynamics, and Materials Conference and Exhibit, 3-8 April 2000, Atlanta, Georgia, AIAA 2000-1636. 